\title{
To the theory of electromagnetic radiation by fast moving body
}

\author{
S. O. Gladkov \\ Moscow Aviation Institute (MAI), National Research University, Rusia
}

\begin{tabular}{l} 
Article Info \\
\hline Article history: \\
Received Aug 1, 2019 \\
Revised Feb 2, 2020 \\
Accepted Mar 13, 2020 \\
\hline Keywords: \\
Electromagnetic radiation \\
The electrons of conductivity \\
The intensity of radiation \\
The lienar-viechert's potentials \\
Wave length
\end{tabular}

\begin{abstract}
The problem of finding out the connection between the flight speed of a metal object and the length of electromagnetic radiation that accompanies its movement has been solved. The calculations are based on the application of Lienar-Vihert's potential theory. Based on the assumption of the dipole mechanism of radiation, the intensity of electromagnetic radiation was calculated and its distribution by coordinates was found. Correlation between wavelength of radiation $\lambda$ and speed of movement $\mathrm{v}$ is established. It is shown, that $\lambda \square \frac{1}{\sqrt{\mathrm{v}}}$.
\end{abstract}

Copyright () 2020 Institute of Advanced Engineering and Science. All rights reserved.

\section{Corresponding Author:}

Gladkov Sergey O,

Moscow Aviation Institute (MAI),

National Research University,

Volokolamskoe shosse, 4. 125993, Moscow, Russia.

Email: sglad51@mail.ru

\section{INTRODUCTION}

The question on which we would like to stop now refers to the general theoretical problems of the thermodynamic theory of elasticity, which have an important applied value. This is the problem of calculating the intensity and power of electromagnetic radiation (abbreviated to EMR), which is an inherent property, always manifesting itself as a result of heating bodies when they move with high speeds in the viscous continuum. The problem is to clarify the relationship between the speed of the bodies $\mathbf{v}$ And the wavelength of EMR $\lambda$, which is a natural consequence of the movement of the conductive body. To solve this problem it's simply introduce the following algorithm of the decision.

1) Calculating the heating temperature as a function of the speed of movement. 2) Finding the connection between temperature and deformation. 3) Establishing a connection between the strain vector and the electric field generated by it, and calculating the potentials of the Lienar - Vihert, appearing outside the body as a result of heating. 4) Analytical assessment of the intensity and power of the resulting EMR. 5) Calculating the relationship between the wavelength of this radiation and the speed of movement. The planned course of the solution of the problem allows us to easily cope with the task, and to establish the dependence that interests us $\lambda(\mathrm{v})$. In the accordance with the planned solution of the problem we start from the first point.

\section{CALCULATING THE HEATING TEMPERATURE AS A FUNCTION OF THE SPEED OF MOVEMENT}

To solve this part of the task we need introduce two parameters such as entropy $S$ of the body and the dissipative function of the continuum $\dot{Q}$. I.e. we have as it's shown in [1-6]. 


$$
T \dot{S}=\dot{Q}
$$

where $T$ - is the temperature. Because we have of the equality $\dot{Q}=-\mathbf{F}_{f r} \cdot \mathbf{V}$, where $\mathbf{F}_{f r}$ - is the resistance force directed against the velocity vector of the body, we can easily estimate the heating temperature from the left side of the (1). Indeed, in the case when the considering particles are satisfactory the Fermi - Dirac's statistics, the entropy can be determined due to the correlation [1].

$$
S=-\sum_{\mathbf{p}}\left(1-n_{p}\right) \ln \left(1-n_{p}\right)+n_{p} \ln n_{p}
$$

where $n_{p}-$ is the distribution function.

The time derivative from of the functionality (2) will be

$$
\dot{S}=\sum_{\mathbf{p}} \dot{n}_{p} \ln \left(n_{p}^{-1}-1\right)
$$

Taking into account (1) we find from here that

$$
\dot{Q}=T \sum_{\mathbf{p}} L\left(n_{p}\right) \ln \left(n_{p}^{-1}-1\right)=C_{P} \dot{T}
$$

where $C_{P}$ - is the isobaric heat capacity of the body, and $L\left(n_{p}\right)-$ is the electron collision integral.

Due to the quasi-classical kinetic equation method for nonequilibrium processes and several research studies [7-16] have suggested that in the case of non compressible liquid the dissipative function from the expression (4) will be following,

$$
\dot{Q}=\frac{\eta}{2} \int_{V}\left(\frac{\partial \mathrm{v}_{i}}{\partial x_{k}}+\frac{\partial \mathrm{v}_{k}}{\partial x_{i}}\right)^{2} d V
$$

where $\mathbf{v}-$ is the velocity of the hydrodynamic flux, $\eta-$ is the dynamic viscosity.

To concretize the solution of the problem, we will assume that the body is a ball of the radius $a$. In this case the dissipative function can be easily computed using the Stokes solution, the details the fierstly presented in 1998 [3]. As a result we have

$$
\dot{Q}=6 \pi \eta a \mathrm{v}^{2}
$$

And with the accordance to expression (4) we find

$$
\int_{V} c_{P} \dot{T} d V=6 \pi \eta a \mathrm{v}^{2}
$$

where $c_{P}-$ it the isobaric heat capacity of the unit volume of the ball, $V=\frac{4 \pi a^{3}}{3}-$ is the ball volume.

Assuming that the speed $\mathrm{v}$ changes over time due to uneven movement of the ball in the viscous medium, we immediately find the desired solution, describing the relationship between the temperature of the surface of the body and the speed of the movement.

$$
\delta T=T-T_{0}=\frac{9 \eta}{2 c_{P} a^{2}} \int_{0}^{t} \mathrm{v}^{2}(t) d t
$$

where $T_{0}-$ is the temperature of the ball at the initial time $t=0$, that we consider the moment of his entry into the viscous continuum. 


\section{CALCULATION OF DEFORMATIONS OF INTERNAL POINTS OF A SPHERE, OBLIGATORY EFFECT OF HEAT HEATING OF SPHERE SURFACE}

To analyze this part of the problem we need to give some explanations. Really, due to the symmetry of the problem and in the assumption of the uniformity of heating the ball, deformation we will consider purely radial. To find them, we use the basic equation of dynamic elasticity theory. In the accordance with [17] we will present it as,

$$
\Delta \mathbf{u}+\frac{1}{1-2 \sigma} \operatorname{graddiv} \mathbf{u}=\frac{\ddot{\mathbf{u}}}{c_{s t}^{2}}+\frac{K \beta}{G} \nabla T
$$

where $\mathbf{u}-$ is the displacement vector internal points of the body, $\sigma-$ is the Poisson's coefficient, $c_{s t}-$ is the transverse speed of the sound, $K-$ is the full compression ratio, $\beta=\frac{1}{V}\left(\frac{\partial V}{\partial T}\right)_{S}$ - is the temperature expansion coefficient, $G$ - is the shift module.

Appearing on the right side of the (9) the temperature gradient due to the spherical symmetry of the problem, we will replace as a differential expression, i.e. as,

$$
\nabla T=\mathbf{n} \frac{\delta T}{\delta}
$$

where $\mathbf{n}=\frac{\mathbf{r}}{r}-$ is a unit vector in the radial direction to the center of the ball, and $\delta-$ is the small linear size near the surface of the sphere, characterizing the area of the temperature change.

Absolutely similarly we have the right to write the following differential expressions

$$
\Delta \mathbf{u} \approx-\frac{\mathbf{u}}{\delta^{2}}, \text { graddiv } \mathbf{u} \approx-\frac{\mathbf{u}}{\delta^{2}}
$$

Therefore the (9), taking into account (10), will greatly simplify, and take the form

$$
\ddot{\mathbf{u}}+\omega_{0}^{2} \mathbf{u}=-\frac{K \beta c_{s t}^{2}}{G \delta} \mathbf{n} \delta T(t)
$$

where the frequency $\omega_{0}=\frac{c_{s l}}{\delta}$ and the longitudinal velocity of the sound is

$$
c_{s l}=c_{s t} \sqrt{\frac{2(1-\sigma)}{1-2 \sigma}} .
$$

The solution of the (11) can be written as

$$
\mathbf{u}(t)=\mathbf{C} \cos \left(\omega_{0} t+\alpha\right)-\frac{K \beta c_{s t}^{2}}{G \omega_{0} \delta} \mathbf{n} \int_{0}^{t} \delta T\left(t^{\prime}\right) \sin \omega_{0}\left(t-t^{\prime}\right) d t^{\prime}
$$

where $\mathbf{C}$ and $\alpha$ are the integration constants.

Without restriction of generality we have assume, that $\mathbf{C}=0$. Then, substituting here the explicit decision from (9), as a result of simple integration, we shall find for the vector of thermal deformations

$$
\mathbf{u}(t)=\frac{9 \eta K \beta c_{s t}}{c_{P} G \omega_{0} a^{2}} \mathbf{n} \int_{0}^{t} \mathrm{v}^{2}\left(t^{\prime}\right) \sin ^{2}\left(\frac{\omega_{0}\left(t-t^{\prime}\right)}{2}\right) d t^{\prime}
$$




\section{CALCULATION OF THE ELECTRIC AND MAGNETIC FIELDS GENERATED BY THE} INNER DEFORMATION OF THE BALL

To solve this part of the task, we will start with an electric field. Provided that internal deformations within the sphere lead to the appearance of an electric field similar to that shown, for example in refs. $[18,19]$ the electrical field generated by the deformation should be defined as

$$
\mathbf{E}^{\prime}=\frac{\rho_{b}}{\rho_{q}} \ddot{\mathbf{u}}
$$

where $\rho_{b}=m n-$ is the body density, $\rho_{q}=e n-$ is the density of the electric charge, $n-$ is the concentration of charges $e$ with the mass $m$. Therefore, the vector potential $\mathbf{A}^{\prime}$ by virtue of the definition $\mathbf{E}^{\prime}=-\frac{\dot{\mathbf{A}}^{\prime}}{c}$ in the according with (14) is

$$
\mathbf{A}^{\prime}=-c \frac{\rho_{b}}{\rho_{q}} \dot{\mathbf{u}}
$$

And, hence, the magnetic field

$$
\mathbf{H}^{\prime}=\operatorname{rot} \mathbf{A}^{\prime}=-c \frac{\rho_{b}}{\rho_{q}} \operatorname{rot} \dot{\mathbf{u}}
$$

Thus, instead of (14) taking into account the solution (13) for the electric field generated by the thermal deformation, we have

$$
\mathbf{E}^{\prime}=\frac{9 m \eta K \beta \omega_{0} c_{s t}}{c_{P} e G a^{2}} \mathbf{n} \int_{0}^{t} \mathrm{v}^{2}\left(t^{\prime}\right) \cos 2 \omega_{0}\left(t-t^{\prime}\right) d t^{\prime}
$$

For the magnetic field we get

$$
\mathbf{H}^{\prime}=-\operatorname{rot} \frac{9 m \eta K \beta c c_{s t}}{2 c_{P} e G a^{2}} \int_{0}^{v^{2}}\left(t^{\prime}\right) \sin \omega_{0}\left(t-t^{\prime}\right) d t^{\prime}=0
$$

As can be seen from (17) and (18), the contribution will be given only by the component of the electric field and the density of the arising electric current generated by thermal deformations in the accordance with the differential Ohm's law $\mathbf{j}^{\prime}=\mathbf{j}_{0}+\sigma \mathbf{E}^{\prime}$, where $\sigma-$ is the conductivity. Due to the (17) the current density is

$$
\mathbf{j}^{\prime}=\mathbf{j}_{0}+\sigma \frac{9 m \eta K \beta \omega_{0} c_{s t}}{c_{P} e G a^{2}} \mathbf{n} \int_{0}^{t} \mathrm{v}^{2}\left(t^{\prime}\right) \cos 2 \omega_{0}\left(t-t^{\prime}\right) d t^{\prime}
$$

where $\mathbf{j}_{0}=$ en $\dot{\mathbf{u}}$. In the abbreviated form

$$
\mathbf{j}^{\prime}=e n \dot{\mathbf{u}}+\sigma \frac{m}{e} \ddot{\mathbf{u}}=\rho_{q}(\dot{\mathbf{u}}+\tau \ddot{\mathbf{u}})
$$

The expression (20) we received as a result of the Drude's formula in the accordance with $\sigma=\frac{e^{2} n \tau}{m}$, where $\tau-$ is the time of the electron relaxation. 


\section{CALCULATING THE INTENSITY AND POWER OF EMR CAUSED BY INERTIAL MOVEMENT OF CHARGES ALONG WITH THE BODY}

In the non-relativistic approximation vector potential A satisfies the Poisson's equation and according to (20) it can be written that:

$$
\Delta \mathbf{A}=-\frac{4 \pi \mu \rho_{q}}{c}(\dot{\mathbf{u}}+\tau \ddot{\mathbf{u}})
$$

where $\mu-$ is the magnetic permeability of the ball.

The solution of the (21) for the external problem of the mathematical physics i.e. $r \geq a$ leads to a solution as an Lienar-Vihert's potential [1] and in my researches [20-25], but taking into account not a single electron, but their ensemble, the density of the charge distribution in which there is $\rho_{q}$. It means that,

$$
\mathbf{A}=\frac{\mu \sigma \rho_{q} V}{c R}(\dot{\mathbf{u}}+\tau \ddot{\mathbf{u}})
$$

where $R=\left|\mathbf{r}-\mathbf{r}_{0}(t)\right|$ and $\mathbf{r}_{0}(t)-$ is the trajectory of the body movement.

Therefore, the field of the radiation as a result of the moving ball thanks to (22) will be,

$$
\mathbf{E}_{r a d}=-\frac{\dot{\mathbf{A}}}{c}=-\frac{\mu \sigma \rho_{q} V}{c^{2} R}(\ddot{\mathbf{u}}+\tau \dddot{\mathbf{u}})
$$

And the intensity of the radiation with the accordance to (23) is the following,

$$
I=c \frac{\mathbf{E}_{r a d}^{2}}{8 \pi}=\frac{\left(\mu \sigma \rho_{q} V(\ddot{\mathbf{u}}+\tau \dddot{\mathbf{u}})\right)^{2}}{8 \pi c^{3} R^{2}}
$$

It means that the power of radiation will,

$$
W=4 \pi R^{2} I=\frac{\left(\mu \sigma \rho_{q} V(\ddot{\mathbf{u}}+\tau \dddot{\mathbf{u}})\right)^{2}}{2 c^{3}}
$$

Because the frequency $\omega_{0}$ satisfies inequality $\omega_{0} \leq \frac{1}{\tau}$, it can be considered relatively large, and we have the right to neglect the second derivative in time on the left side of the (11). This will result in a significant simplification of the solution (13) and in this case it is easy to find that,

$$
\mathbf{u}(t)=\frac{9 \eta K \beta c_{s t}^{2} \delta}{2 c_{P} G c_{s l}^{2} a^{2}} \mathbf{n} \int_{0}^{t} \mathrm{v}^{2}\left(t^{\prime}\right) d t^{\prime}
$$

Therefore, according to (26) the rate of deformation caused due to the thermal impact will be,

$$
\dot{\mathbf{u}}(t)=\frac{9 \eta K \beta c_{s t}^{2} \delta}{2 c_{P} G c_{s l}^{2} a^{2}} \mathbf{n} v^{2}
$$

Hence,

$$
\ddot{\mathbf{u}}(t)=\frac{9 \eta K \beta c_{s t}^{2} \delta}{c_{P} G c_{s l}^{2} a^{2}} \mathbf{n}(\mathbf{v} \cdot \dot{\mathbf{v}})
$$




$$
\dddot{\mathbf{u}}(t)=\frac{9 \eta K \beta c_{s t}^{2} \delta}{c_{P} G c_{s l}^{2} a^{2}} \mathbf{n}\left(\dot{\mathbf{v}}^{2}+\mathbf{v} \cdot \ddot{\mathbf{v}}\right)
$$

In a very good approximation, if the movement occurs in the field of the gravity, the speed of movement of the body can be imagined as,

$$
\mathbf{v}=\mathbf{v}_{0}+\mathbf{g} t
$$

Then at relatively small times of traffic, when $\Delta t \square \frac{\mathrm{v}_{0}}{g}$ and in a very good approximation $|\ddot{\mathbf{u}}| \square \tau|\ddot{\mathbf{u}}|$ of the solution (24), taking into account (28) we will find,

$$
I=\frac{9 \eta K \beta c_{s t}^{2} \delta}{8 \pi c_{P} G c_{s l}^{2} a^{2}} \frac{\left(\mu \sigma \rho_{q} V\right)^{2}}{c^{3} R^{2}}\left(\mathbf{v}_{0} \cdot \mathbf{g}\right)^{2}
$$

According the power of the radiation will,

$$
W=\frac{9 \eta K \beta c_{s t}^{2} \delta}{2 c_{P} G c_{s l}^{2} a^{2}} \frac{\left(\mu \sigma \rho_{q} V\right)^{2}}{c^{3}}\left(\mathbf{v}_{0} \cdot \mathbf{g}\right)^{2}
$$

\section{DETERMINING THE RELATIONSHIP BETWEEN THE LENGTH OF EMR AND THE SPEED OF BODY MOVEMENT}

In order to answer the question posed, we need to focus on a specific physical assumption that will allow us to establish an appropriate link. In fact, since the internal crystalline structure of the body at the atomic level changes in the conditions of thermal expansion, it is quite obvious that the atom is shifted from the initial state of equilibrium in the radial direction for some small distance $\Delta r$. This is due to the spherical symmetry of the problem to be solved and the condition of the uniformity of the heat load, if the outer shell of the atom is $Z e$, the arising dipole moment will be $d_{r}=Z e \Delta r$. This means that the both the dipole radiation and the radiation (31) must be equal. I.e. the dipole radiation and above calculated with the accordance of the formulae (31) and (32) are equal because the other in the nature we have no. The sounded assertion seems to be quite reasonable within the framework of the theory of the dipole radiation, well stated in the [1]. Adhering to this theory, we will present the vector potential, bound to the dipole moment in the form of:

$$
\mathbf{A}^{\prime \prime}=\frac{\mu n V \dot{\mathbf{d}}}{c R}
$$

Then the outer field of radiation generated by these dipole moments will be

$$
\mathbf{E}^{\prime \prime}=-\frac{\mu n V \ddot{\mathbf{d}}}{c^{2} R}
$$

The intensity of the radiation then,

$$
I^{\prime \prime}=\frac{\mu^{2} n^{2} V^{2}|\ddot{\mathbf{d}}|^{2}}{8 \pi c^{3} R^{2}}
$$

and the power will

$$
W^{\prime \prime}=\frac{\mu^{2} n^{2} V^{2}|\ddot{\mathbf{d}}|^{2}}{2 c^{3}}
$$


Assuming that $\mathbf{d}=\mathbf{d}_{0} e^{-i \omega t}$, where the frequency of the monochromatic EM wave is $\omega=k c$, where the wave vector $k=\frac{2 \pi}{c}$, from the expression (36) we get,

$W^{\prime \prime}=\frac{\mu^{2} n^{2} V^{2} \omega^{4} \mathbf{d}_{0}^{2}}{2 c^{3}}$

Equating expressions (37) and (32) we find,

$$
\frac{\mu^{2} n^{2} V^{2} \omega^{4} \mathbf{d}_{0}^{2}}{2 c^{3}}=\frac{9 \eta K \beta c_{s t}^{2} \delta}{2 c_{P} G c_{s l}^{2} a^{2}} \frac{(\mu \sigma e n V)^{2}}{c^{3}}\left(\mathbf{v}_{0} \cdot \mathbf{g}\right)^{2}
$$

After all the cuts will finally have for the frequency of EMR,

$$
\omega=\sqrt{\frac{3 \sigma e \sqrt{\eta K \beta \delta}}{a \sqrt{c_{P} G}} \frac{c_{s t}}{c_{s l}} \frac{\left(\mathbf{v}_{0} \cdot \mathbf{g}\right)}{d_{0}}}
$$

It means that the wavelength of the radiation will depend on the velocity of the body as a function,

$$
\lambda=\frac{\xi}{\sqrt{\left(\mathbf{v}_{0} \cdot \mathbf{g}\right)}}
$$

where the physical coefficient

$$
\xi=\frac{2 \pi c}{\sqrt{\frac{3 \sigma e \sqrt{\eta K \beta \delta}}{a d_{0} \sqrt{c_{P} G}}} \frac{c_{s t}}{c_{s l}}} .
$$

That was required to show. Note as we can see from determination of the parameter $\xi$, it includes all physical properties of both: body and continuum. It means that we can very easy to estimate the numerical value of the parameter $\xi$ and calculate of the wavelength $\lambda$ in the accordance of formula (39).

\section{RESULTS AND DISCUSSION}

As a result of the study in this section it is worth noting that for the first time it was possible to find a connection between the speed of movement of a metal object and the electromagnetic wave length it emits. The fundamental point of the built theory is the condition of uneven movement of the body, which always takes place in the conditions of acceleration and braking. This means that for accurate navigation devices it is always easy to record of the start and the speed of the body.

\section{CONCLUSION}

In the conclusion of the work we will pay attention to a number of the main results of the above constructed theory. It is shown that any moving with the speed of $\mathrm{v}_{0}$ ball (as an as the body of another form) will emit monochromatic EM wave with the wavelength in the accordance to the dependence (39). The wavelength of this radiation should decrease with the growth of the speed of the movement. 


\section{ACKNOWLEDGEMENTS}

The author expresses sincere gratitude to all participants of the theoretical seminar for useful remarks and discussions of the results of the work.

\section{REFERENCES}

[1] L. D. Landau and E. M. Lifshitz, "Statistical Physics," Pergamon Press, vol. 5, 2001.

[2] G. K. Schenter and R. L. Liboff, "Quasiclassical Kinetic Equation for Charge-Carrier Transport in a Semiconductor," Journal of Applied Physics, vol. 62, pp. 177-186, 1987.

[3] S. O. Gladkov, "Microscopic Derivation of Darcy's Law," Russian Physics Journal, vol. 41, pp. 969-974, 1998.

[4] S. O. Gladkov, "On the Connection of Joule - Thompson's Coefficient with Dissipation Properties of Filtrating Media," The European Physical Journal E, vol. 10, no. 2, pp. 171-174, 2003.

[5] V. G. Baryakhtar and A. G. Danilevich, "Dissipative Function on a Ferromagnet and the Theory of Onsager's Kinetic Equations,” Low Temperature Physics, vol. 41, no. 10, pp. 997-1000, Oct 2015.

[6] H. Goldstein, "Classical Mechanics," $2^{\text {nd }}$ edition, MA, Addison-Wesley, 1980.

[7] G.O. Balabanyan. "Kinetic Equations for Nonequilibrium Quantum Systems. Physics Letters A, vol. 48A, pp. 275281, April 1974.

[8] S. O. Gladkov, "The Kinetics of Nuclear Magnetically Ordered Systems," Physics Reports, vol. 182, no. 4-5, pp. 211-364, April 1989.

[9] A. Rangel-Huerta, R.M. Velasco. "Generalised Bulk Viscosity for Enskog Gases". Journal of Non-Equilibrium Thermodynamics, vol. 21, no. 4, pp. 321-329, Apr. 1996

[10] M. Groppi, et al., "Generalized Quasi-Classical Boltzmann Equation for Homogeneous Reacting Gases," Transport Theory and Statistical Physics, vol. 32, no. 5-7, pp. 567-586, May 2003.

[11] V.P. Belavkin, V.N. Kolokoltsev. "On a General Kinetic Equation for Many-Particle System with Interactio, Fragmentation and Coagulation”, Proceedings of the Royal Society A, vol. 459 (2031), pp.727-748, Mar. 2003.

[12] V. Spicka, et al., "Long and Short Time Quantum Dynamics: II. Kinetic Regime," Physica E: Low-dimensional Systems and Nanostructures, vol. 29, no. 1-2, pp. 175-195, Jan. 2005.

[13] V.I. Mazhukin, A.V. Mazhukin, O. Koroleva. "Optical Properties of Electron Fermi-Gas of Metals at Arbitrary Temperature and Frequency”, Laser Physics, vol. 19, no. 5, pp. 1179-1186, May 2009.

[14] I. Paidarova, V. Paidar, P. Durand. "A Simple Model of Dissipative Processes in Ferroelastics", Solid State Phenomena, vol. 172-174, pp. 1189-1194, June 2011.

[15] E. Arrigoni, M. Knap, W. Linden. "Nonequilibrium Dynamical Mean-Field Theory”, Physical Review Letters, vol. 110 (8): 086403, Feb. 2013.

[16] V. Spicka, et al., "Electron System out of Equilibrium: Nonequilibrium Green's Functon Approach," International Journal of Modern Physics B, vol. 28, no. 23, p. 1430013, 2014.

[17] L. D. Landau and E. M. Lifshitz, "Fluid Mechanics," Pergamon Press, vol. 6, 2002.

[18] L. D. Landau and E. M. Lifshitz, "Theory of elasticity," Pergamon Press, vol. 7, 2004.

[19] L. D. Landau and E. M. Lifshitz, "Electrodynamics of continuous media," Pergamon Press, vol. 8, 2004.

[20] S. O. Gladkov, "Theory of Absorption of Electromagnetic Radiation Energy by Fins Metal Particles," Journal of Applied Mechanics and Technical Physics, vol. 52, no. 2, pp. 159-168, Feb. 2011.

[21] S. O. Gladkov, "Intensity of Electromagnetic Energy Radiation by a Quiscent Ferromagnetic Spherical Particle Placed in a Permanent Magnetic Field,” Technical Physics, vol. 60, pp. 1082-1085, July 2015.

[22] S. O. Gladkov and S. B. Bogdanova. "A Rotating Ferromagnetic Sphere as a Source of Long-Wavelength Electromgnetic Radiation,” Journal of Communications Technology and Electronics, vol. 62, pp. 740-749, 2017.

[23] S. O. Gladkov and S. B. Bogdanova. "On the Intensity of Radiation of an Electromagnetic Field by a Rotating Ferroelectric Sphere,” Russian Physical Journal, vol. 61, no. 1, pp. 102-108, Jan 2018.

[24] M. Salazar and G. A. P. Alcazar, "The Dissipative Function in Magnetic Systems," Physica A: Statistical Mechanics and its Aplications, vol. 517, pp. 337-340, Mar 2018.

[25] S. O. Gladkov, "To the theory of electromagnetic fields distributions for Lienar - Vihert's potentials," Engineering Physics", vol. 19, no. 8, pp. 3-10, Aug 2018.

\section{BIOGRAPHY OF AUTHORS}

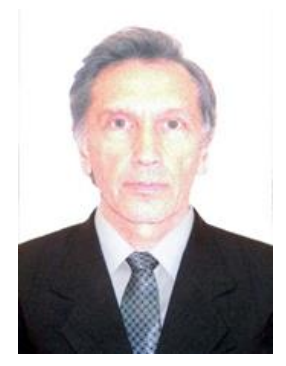

Gladkov Sergey Oktyabrinovich, Dr. of Physical and Mathematical Science, Professor of Theoretical Physics. Have near 200 science publications in WoS and Scopus Journals. The joint of member of board Ed. Journal Complex System. Research ID: U-1072-2018.

Research gate_Gladkov S.O. 